\title{
Method to Calculate Fluctuations in the Strength of Radio Noise Emitted from Electric Railway Systems
}

\author{
Kunihiro KAWASAKI \\ Senior Researcher, Laboratory Head, \\ Telecommunications Systems Laboratory, Signalling and Telecommunications Technology Division

\begin{abstract}
In order to identify the characteristics of radio noise emitted from the operation of electric railway systems and evaluate the effects of countermeasures to reduce it, timeand cost-intensive measuring tests are currently necessary. To reduce the amount of time and the costs involved, our institute started a study in 2005 to establish a method of calculating the strength of noise radiated from electric railway systems. As a result of this study, a basic method of determining trends in fluctuations of the strength of radio noise emitted from running electric vehicles has been developed using the moment method. This paper outlines the characteristics of radio noise emitted from electric railway systems and reports on the results of a basic study to establish the calculation method.
\end{abstract}

Keywords: electric traction, radio interference, electromagnetic compatibility, simulation

\section{Introduction}

The IEC 62236-2 international electromagnetic compatibility (EMC) standard, which defines measuring methods and emission limits for radio noise emitted from railway systems, has already been established by the International Electrotechnical Committee (IEC). In Japan, many on-site tests have been carried out in accordance with this standard to check EMC in environments along railway lines or to ascertain the characteristics of radio noise and evaluate the effects of countermeasures to reduce it. However, these on-site tests require large amounts of time and money, so there is a need for effective methods to calculate the strength of radio noise from railway systems without this kind of testing. Our institute has been conducting basic research toward the establishment of such a method since 2005 .

\section{Radio noise emitted from electric railway systems}

A variety of electric and electronic equipment and apparatus are installed in electric railway systems, as shown in Fig. 1. Generally speaking, such systems have two major noise emission sources. One is arc discharge caused by contact breaks between pantographs and trolley wires, and the other is the harmonic current included in feeding and return current.

Arc discharge between pantographs and trolley wires used to be the major noise emission source before VVVF control vehicles appeared. The phenomenon stems from contact breaks, and radiates high-level radio noise in the frequency range of the VHF television broadcasting band (around $100 \mathrm{MHz}$ to $200 \mathrm{MHz}$ ). Accordingly, various countermeasures to reduce the level of radio noise caused by contact breaks were developed and installed on Shinkansen and other vehicles $[1,2]$. Thanks to these countermeasures, the strength of radio noise resulting from arc discharge has now been reduced, and emission phenomena caused by contact breaks occur less fre-

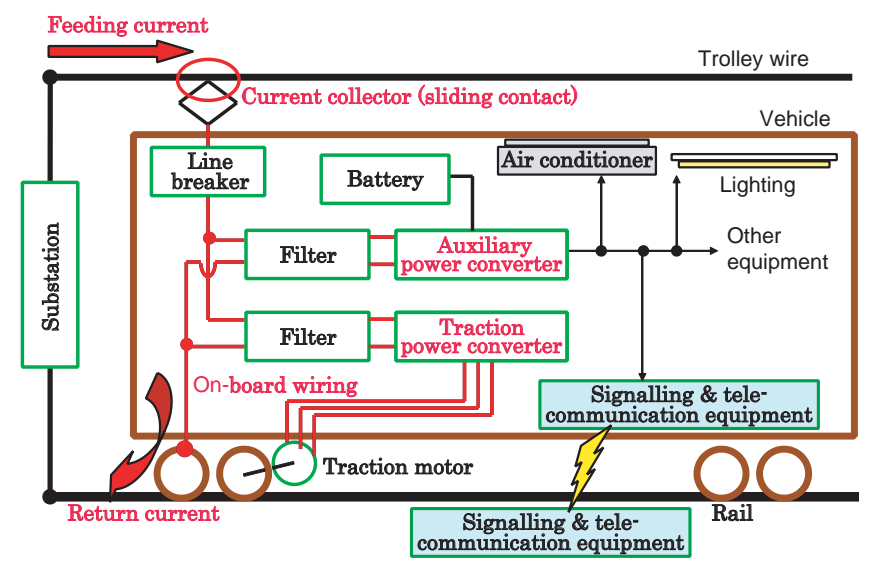

Fig. 1 Outline of an electric railway system (a DC feeding system)

quently.

After the appearance of VVVF control cars in the late 1980 s, the strength of radio noise generated by traction power inverters on vehicles increased in the frequency range below around $10 \mathrm{MHz}$.

An example of temporal variations in field strength during a measuring test based on IEC 62236-2 high-speed running train testing is shown in Fig. 2, and Fig. 3 shows an example of a test setup based on IEC 62236-2 in Japan. In Fig. 2, the time in seconds is plotted on the horizontal axis. Zero seconds is the time at which the head of the train passes the measuring antenna. The relative field strength in $\mathrm{dB}$ is plotted on the vertical axis. The green line indicates field strength at a frequency of 100 $\mathrm{MHz}$, and the blue and red lines indicate field strength at the frequencies of $0.1 \mathrm{MHz}$ and $1 \mathrm{MHz}$ respectively. Zero $\mathrm{dB}$ is the average ambient field strength at each frequency measured during the site test. Usually, in the frequency range below about $10 \mathrm{MHz}$, the maximum radio noise level is recorded when the train is far from the measuring point as shown in Fig. 2, and in the frequency 


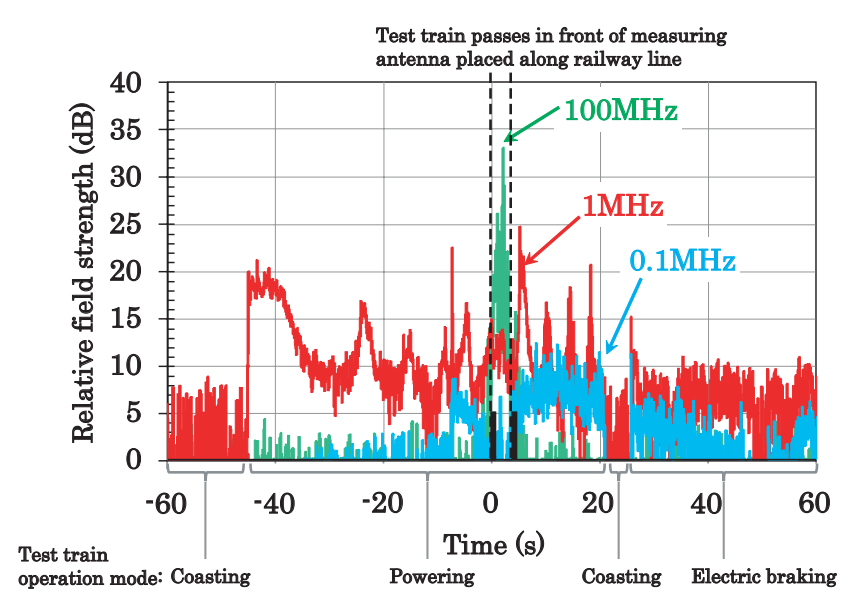

Fig. 2 Example of temporal variations in field strength during a measuring test based on IEC 62236-2

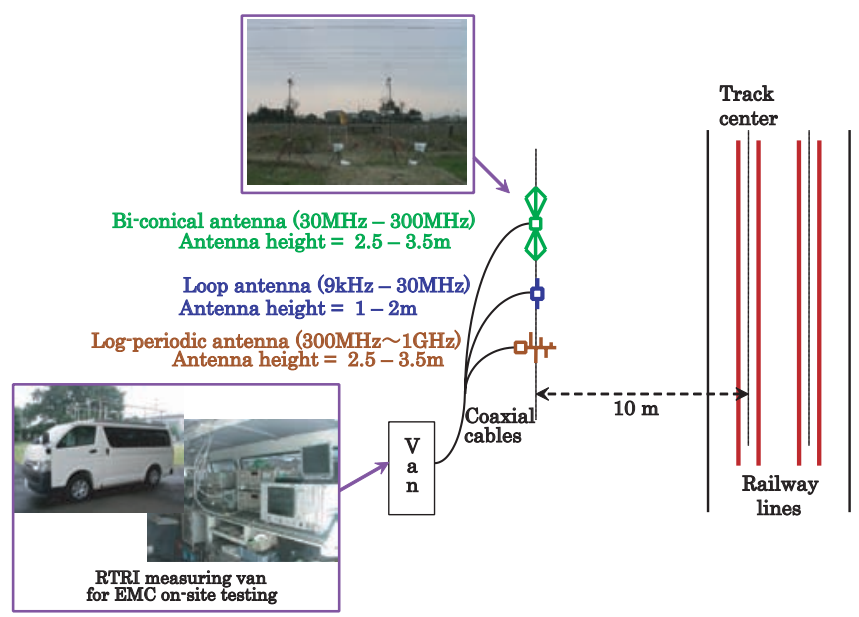

Fig. 3 Example of a measuring test setup based on IEC 62236-2 in Japan

range above about $10 \mathrm{MHz}$, it is recorded when the train passes the measuring point.

Moreover, the strength of radio noise is directly related to the operation mode of traction units (powering, coasting, electric braking) in the frequency range from $9 \mathrm{kHz}$ to about $10 \mathrm{MHz}$.

\section{Modeling of emission phenomena}

\subsection{Basic concepts}

Temporal variations in the strength of radio noise radiating from railway systems in areas along railway tracks are very complex, and the characteristics differ depending on the measuring frequency. A model to represent such a complex phenomenon and calculate the strength of radio noise becomes quite complicated.

Accordingly, we decided to make a basic representation that can calculate the relative trends of fluctuations in the strength of radio noise as the first stage of modeling. The ideal would be to construct a model that incorporates all components found in railway systems, but to minimize the scale of the model and reduce computational effort, the following points were assumed as modeling preconditions:

- The feeding system is the simplest DC type

- The railway system consists of four components: a substation, a straight single trolley wire, a single traction vehicle, and a single straight rail.

- The single traction vehicle has a simple pantograph and return circuit and one or two radio noise generators. In reality, radio noise contains various frequency components and its strength varies temporally, but in this simple model, the radio noise generators act as single-frequency sine-wave oscillators.

\subsection{Consideration of practical numerical analysis methods}

There are many numerical methods for analyzing the radiation of electromagnetic waves, including the Finite Difference Time Domain (FDTD) method, the Finite Element Method (FEM) and the moment method. A technique for analysis that treats the components of railway systems as antenna elements has been researched, but no usable and practical technique to calculate the strength of noise radiated from operating railway systems has yet been established.

Accordingly, we modeled a whole electric railway system as a large antenna and calculated the emission strength from the antenna model using the moment method. This is a kind of Boundary Element Method (BEM), and is used for analyzing the behavior of various types of antenna [3].

An example of an antenna model based on the idea described above is shown in Fig. 4, which shows that all components are expressed as a group of metallic wires, and two emission sources are placed on the antenna model. The emission source placed under the body acts as a noise source due to the harmonic current generated by the traction converter, and the other source placed on the sliding contact acts as a noise source due arc discharge. Vehicle running is simulated by changing the distance between the substation and the vehicle. That is, $L_{s}$ and $L_{e}$ in Fig. 4 are changed to satisfy the following equation in order to indicate the position of the vehicle in

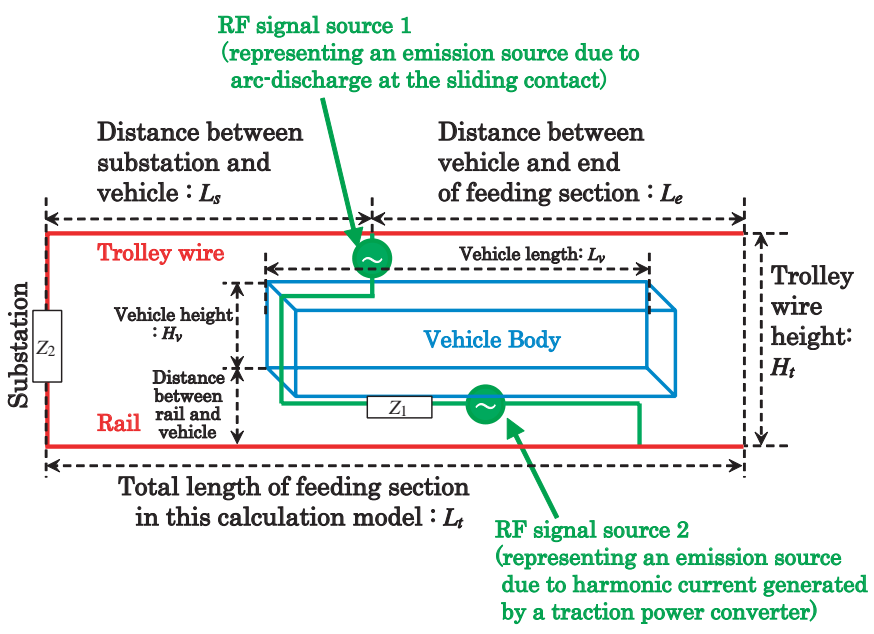

Fig. 4 Example of an antenna model 
the model.

$$
L_{t}=L_{s}+L_{e}
$$

Wiring installation on the vehicle is modeled as a single wire in Fig. 4 , but of course it is possible to construct a model in more detail. $Z_{1}$ and $Z_{2}$ in Fig. 4 indicate lumped parameter elements such as reactors, capacitors and registers, which act as filters or resonators.

Emission sources can also be placed at arbitrary positions on antenna elements.

\subsection{Calculation results}

Figure 5 gives some examples of calculation results for radio frequency currents in the trolley wire of the antenna model described in Fig. 4, and shows the radio frequency current distribution in the wire (frequency = $1 \mathrm{MHz}$ ). These results are obtained using Numerical Electromagnetic Code (NEC) - an antenna modeling program [4]. In this case, we set $\mathrm{L}_{\mathrm{t}}$ to $2,020 \mathrm{~m}$ and $\mathrm{H}_{\mathrm{t}}$ to $5 \mathrm{~m}$, and the relative vehicle position is plotted on the horizontal axis. Zero $\mathrm{km}$ is the point at which the vehicle is in front of the measuring antenna mounted on the ground. The vehicle starts at the substation (relative vehicle position $=-1 \mathrm{~km}$ ) and moves away from it. The picture of the vehicle in Fig. 5 is just a representation, so doesn't show the actual size of the vehicle model. The radio frequency current in milliamperes is plotted on the vertical axis. Figure 5 (a) shows the results for a case where the distance between the substation and the vehicle ( $L_{s}$ in the model) is $0.5 \mathrm{~km}$, (b) shows the results for $L_{s}=1 \mathrm{~km}$, and (c) shows the results for $L_{s}=1.5 \mathrm{~km}$.

It can be seen from Fig. 5 that the radio frequency current distribution in the trolley wire changes depending on the vehicle position. These variations are thought to be determined by the wavelength of the radio frequency current and the characteristics of the large loop antenna.

Next, Fig. 6 shows an example of the calculation results for relative field strength at the position of a measuring antenna mounted on the ground along the railway line. These results are obtained under the conditions of $L_{t}=5,020 \mathrm{~m}$ and $H_{t}=5 \mathrm{~m}$. The measuring antenna (i.e., the observing point) is positioned at $L_{s}=2,500$ $\mathrm{m}$, and the lateral distance to the rail is $10 \mathrm{~m}$. We assumed that the emission source placed under the vehicle body generates a radio frequency current with a frequency of $0.1 \mathrm{MHz}$, and the other emission source placed at the sliding contact generates a radio frequency current with a frequency of $100 \mathrm{MHz}$. Figure 6 (a) shows the results for the frequency of $0.1 \mathrm{MHz}$, and (b) shows the results for that of $100 \mathrm{MHz}$. The relative vehicle position is plotted on the horizontal axis, and the relative field strength in $\mathrm{dB}$ is plotted on the vertical axis. The red dots represent the calculated values, and the green lines show the values measured under conditions similar to those used for the calculation.

Figure 6 shows that, even though the calculation model is very simple, the trends of fluctuations in the strength of radio noise can be identified, and that the method of modeling an electric railway system as a large antenna and analyzing it using the moment method is

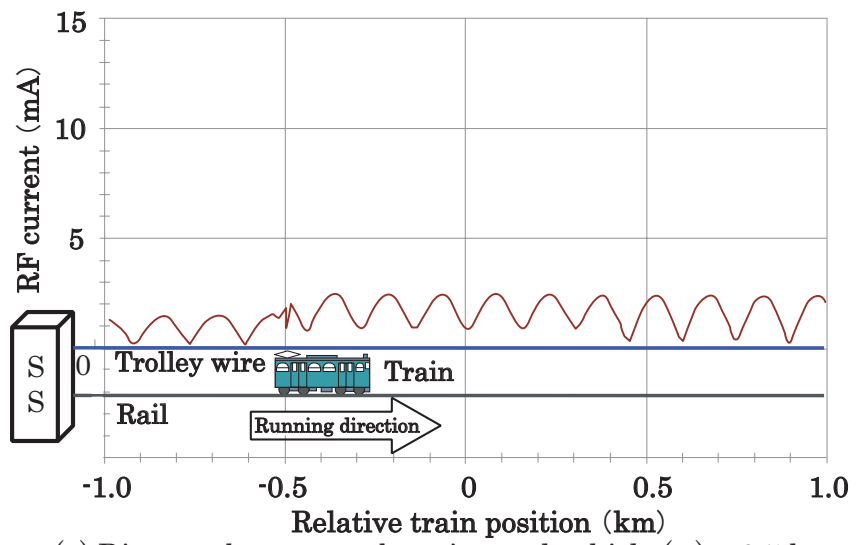

(a) Distance between substation and vehicle $\left(L_{S}\right)=0.5 \mathrm{~km}$

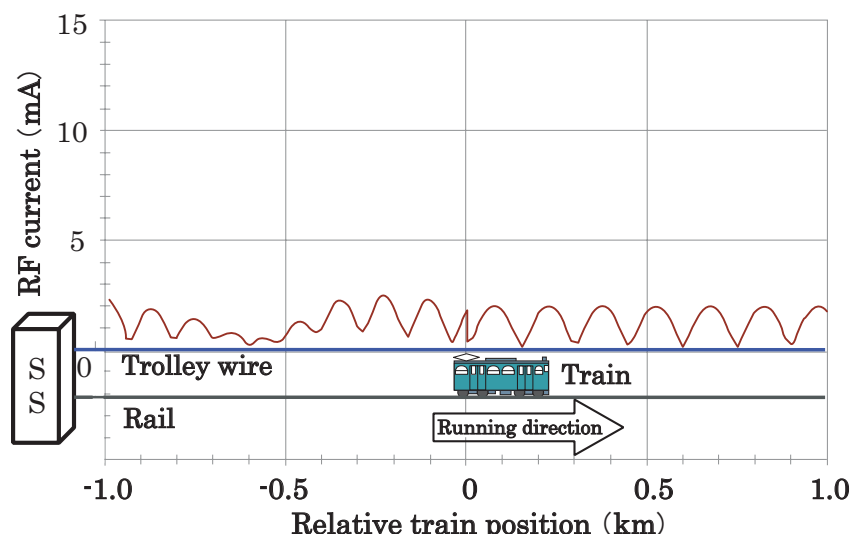

(b) Distance between substation and vehicle $\left(L_{s}\right)=1.0 \mathrm{~km}$ (In this case, the vehicle is just in front of the measuring antenna mounted on the ground along the railway line.)

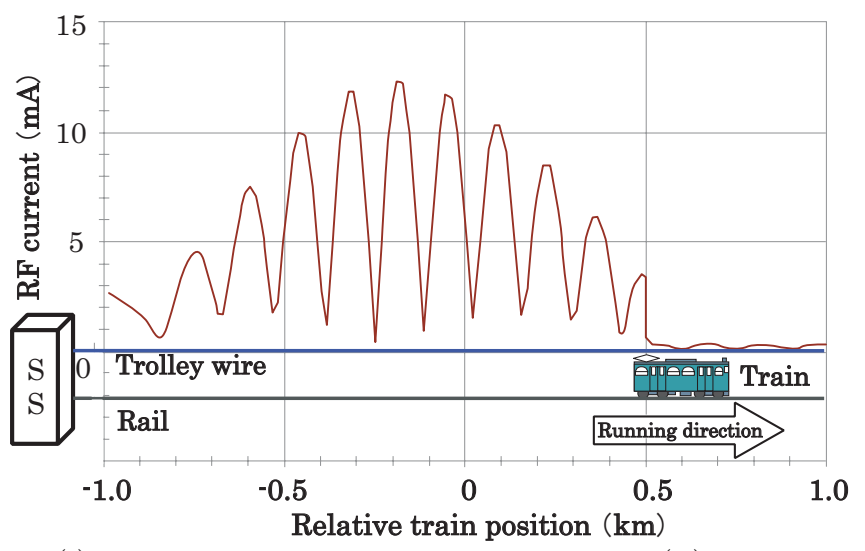

(c) Distance between substation and vehicle $\left(L_{S}\right)=1.5 \mathrm{~km}$

Fig. 5 Example of calculation results for radio frequency current distribution in trolley wire (Frequency of current $=1 \mathrm{MHz}$; the emission source is under the body of the vehicle)

applicable to the calculation of fluctuations in the strength of radio noise radiated from a railway system.

However, the calculated values do not agree with the measured values. The reasons why the calculated results are larger are as follows:

- The power generated by the emission source in this model is higher than that of actual radio noise sources.

- The attenuation of radiated radio noise due to 


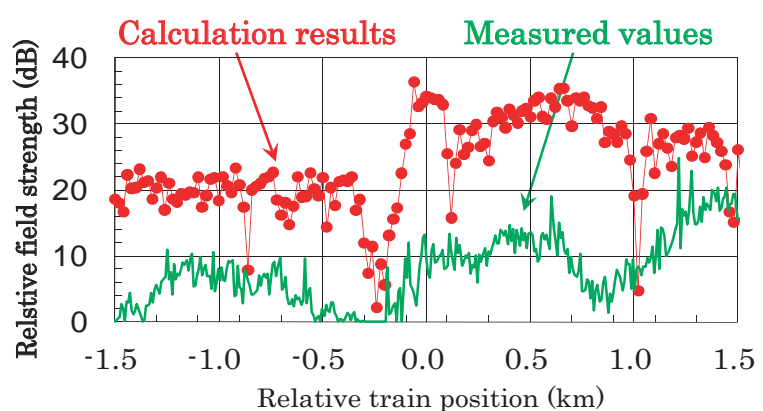

(a) Frequency $=0.1 \mathrm{MHz}$

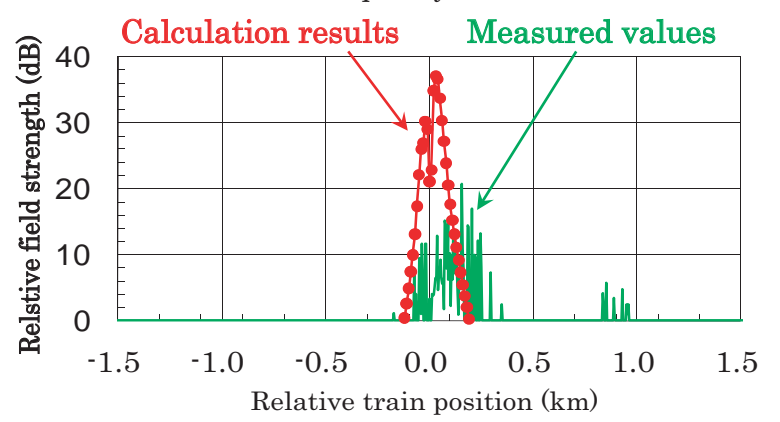

(b) Frequency $=100 \mathrm{MHz}$

Fig. 6 Example of the results of comparison between calculated and measured values

shielding and reflection in the propagation path is omitted.

\section{Application of the method}

The method described in this paper cannot yet be practically applied to calculate absolute strength values. However, it may be possible to use it in predicting the relative effects of countermeasures to reduce radio noise levels under certain conditions. For example, the method can be applied to predict whether any effect from onboard EMI filters can be expected.

Figure 7 shows an example presuming the effect of countermeasures using this method. It shows the results of calculating the radio frequency current with a frequency of $1 \mathrm{MHz}$ distributed in the contact wire with the same model and under the same conditions as the case shown in Fig. 5 (c). The red line represents the results when no countermeasures are introduced, and duplicates the line for the calculation results shown in Fig. 5 (c). The blue line represents the calculated results for the case where a reactor is installed at the position indicated in Fig. 7, which shows that the radio frequency current in the trolley wire with the reactor installed is lower than that in the case without installation. From these calculation results, we can predict that reactor installation will be effective in reducing emission levels.

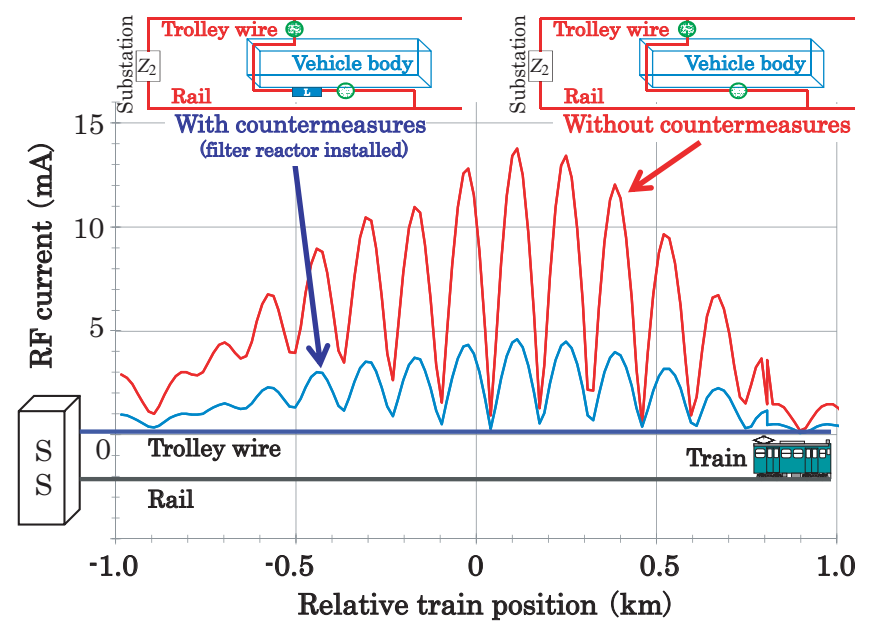

Fig. 7 Example of the proposed method's application

\section{Conclusions}

This paper describes a method for modeling an electric railway system as a large antenna and analyzing it using the moment method. Trial application of this technique showed that fluctuations in the strength of radio noise radiated from a railway system can be simulated with minimal calculation. The method is therefore expected to be practical as a way of calculating radio noise emissions from electric railway systems in the future.

Our institute plans to improve the model and the method in order to obtain more accurate calculation results within a couple of years. We have already started to build a more detailed antenna model that mirrors actual railway systems as closely as possible, and are making efforts to improve models of emission sources to simulate actual noise sources.

\section{References}

[1] T. Kawamura, Y. Yoshida, "Radio Disturbance Wave due to High-Speed Electric Vehicles," Quarterly Report of RTRI, Vol. 33, No. 3, pp. 209-214, 1992.

[2] K. Tsuboi, T. Sagawa, N. Kurita, A. Shindo, "The Effect of Radio Noise Suppressing for Tohoku, Joetsu SHINKANSEN Electric Vehicles," Quarterly Report of RTRI, Vol. 24, No. 1, pp. 44-45, 1983.

[3] R. F. Harrington, "Field Computation by Moment Methods," IEEE Press, 1993.

[4] http://www.nec2.org/

[5] K. Kawasaki, "Basic Study on a Method to Calculate Fluctuations of Strength of Radio Disturbance Wave Emitted from Railway Systems to Railway Side," RTRI Report, Vol. 32, No. 2, pp. 91-96, 2009 (in Japanese). 